\title{
A consensus on the classification of hydrocephalus: its utility in the assessment of abnormalities of cerebrospinal fluid dynamics
}

\author{
Harold L. Rekate
}

Received: 4 August 2011 / Accepted: 9 August 2011

(C) The Author(s) 2011. This article is published with open access at Springerlink.com

\begin{abstract}
Background Between 2009 and 2011 an attempt has been made to develop a consensus on the classification of hydrocephalus. Clinicians and basic scientists who are recognized internationally for their work in hydrocephalus attended working meetings in which the concepts of classification of hydrocephalus were discussed at length. Purpose This review attempts to explain the relevance of a classification scheme based on the point of obstruction to the flow of cerebrospinal fluid to basic science research into the pathophysiology and effects of hydrocephalus. The review is designed to give examples of the value of this classification in analyzing research utilizing animal models. The development of hydrocephalus in the absence of a point of obstruction (true communicating hydrocephalus) is analyzed.

Conclusion Contemporary neuroimaging techniques are now available that can identify the actual point of obstruction to the flow of CSF, if any, which results in the development of hydrocephalus. Such identification may lead to improved ability to analyze animal models used in hydrocephalus research as well as deciding among various treatment options.
\end{abstract}

Keywords Hydrocephalus · Classification .

Pathophysiology $\cdot$ Animal models

H. L. Rekate $(\bowtie)$

Department of Neurosurgery, The Chiari Institute,

Hofstra North Shore-LIJ College of Medicine,

865 Northern Blvd., Suite 302,

Great Neck, NY 110021, USA

e-mail: hrekate@nshs.edu
The classification of hydrocephalus: historical perspective

Research into the basic causes of hydrocephalus began with Dr. Walter Dandy of Johns Hopkins University. As early as 1913 Dandy proposed a classification scheme for hydrocephalus that forms the basis of the accepted system still in use at this time. Dandy had few tools available to him at the time. The first studies in experimental animals related to the injection of a supravital dye into the ventricle and attempting to recover it from the spinal subarachnoid space (SSAS) via lumbar puncture. Based on these studies he classified hydrocephalus as either "communicating" or "obstructive or non-communicating" in nature [1]. The only other technique he could use to study what was happening in experimental animals or in affected individuals was to perform an autopsy. It was not until 1919 that Dandy was able to obtain more precise information about abnormalities of the cerebrospinal fluid (CSF) pathways by the injection of air either by lumbar puncture or its installation within the ventricle. Pneumoventriculography allowed more precise localization of the point of obstruction within the ventricular system as well as demonstrate distorted anatomy within the ventricles such as that caused by tumors in various locations [2]. The findings of Dandy's newly developed tests related to the anatomy of CSF flow did not lead to modification of the classification of hydrocephalus.

The concepts described in the Dandy classification led to advances in the understanding of CSF dynamics and the understanding of the pathogenesis and treatment of hydrocephalus. Based on these concepts, Dandy defined the choroid plexus as the source of the production of CSF and proposed choroid plexectomy for the treatment of this condition. In patients with obstructive hydrocephalus, 
Dandy felt that creating an internal bypass was a likely form of treatment and performed operations in which communication between the third ventricle and the cortical subarachnoid space (CSAS) was created. This was originally done by the resection of one of the optic nerves [1]. Dandy's 1913 classification still forms the basis of the bureaucratic methodology for physician payment in the USA. The International Classification of Diseases, Ninth Revision codes for hydrocephalus carry the same words as did Dandy's work except for the very recent addition of the third category of Idiopathic Normal Pressure Hydrocephalus [3].

Several authors have attempted to develop more contemporary classification schemes, but none have served to improve the understanding of hydrocephalus and until now there has been no consensus as to a more contemporary classification scheme. Raimondi decided to use the translation of the term hydrocephalus as "water head" to produce a classification that included all conditions in which there were more than normal amounts of water within the intracranial compartment and thus included forms of cerebral edema, brain atrophy and intracranial cysts as well as forms of hydrocephalus. This classification is now only quoted in its historical context [4].

Mori with the encouragement and support of the Japanese ministry of health performed a study of various forms of hydrocephalus in Japanese children from many centers throughout Japan. The purpose of this study was to define "intractable hydrocephalus." By this he meant which forms of hydrocephalus do not respond to treatment. In which of these patients is treatment futile [5]? BeniAdani and her colleagues recognized that there was little consensus as to how one selects patients, particularly babies as to whether their hydrocephalus should be treated with a shunt or with an endoscopic third ventriculostomy. It was their intention that the proposed classification would serve to rigorously study the outcome of various treatments on various forms of hydrocephalus so that one would have a better idea as to whom to offer third ventriculostomy, and they presented the data from Israel related to these criteria [6].

Studying the relationship between what is happening in the developing brain and the pathologic processes that were occurring at that point in time led Oi to propose a classification of hydrocephalus that reflected the effect of brain distortion on the fetus and infant. $\mathrm{He}$ discussed its usefulness in the prediction of outcome in babies born with hydrocephalus of various causes. Early prediction of outcome is a very important issue, and the ability to define that outcome in the early mid-trimester would represent a major advance. Studies seem to be ongoing [7].

\section{A consensus on the classification of hydrocephalus}

In 2000 I was asked by Dr. David McLone to write a chapter on the classification of hydrocephalus for the fourth edition of Pediatric Neurosurgery: Surgery of the Developing Nervous System [8]. I had been working with the School of Engineering at Case Western Reserve University on the application of engineering principles to the study of ventricular volume regulation. This process involved the use of a mathematical model which attempted to predict what would happen to the volume of the ventricles and the pressures within those compartments produced by creating resistance elements in the pathways between the CSF compartments. Previous work on such a model was reviewed and particularly the theoretical discussion by Spertell regarding brain viscoelasticity [9]. Our multicompartmental model led to testable hypotheses which could explain what would happen if the sagittal sinus pressure was raised or what would happen if one created a resistance element at the basal cisterns. Figure 1 is a demonstration of the CSF system as interacting compartments each with its own pressure and volume related to the flow of CSF [10,11]. Based on this diagram experiments could be performed on animal models of hydrocephalus that would challenge the results of the mathematics from the mathematical model. The diagram led to the need to measure the pressures and volumes in the individual compartments and to attempt to define the resistances between the various compartments. The result of this thought process became a circuit diagram of the CSF pathway as a hydraulic analog of an electrical circuit [1214]. Figure 2 is an artist's rendering of this circuit diagram analogous to an electrical circuit. Visualizing the flow of CSF in this way serves as a template to assess the possible treatment options that are available to treat an individual patient. What followed has been a 30-year study of hydrocephalus based on this model. It became evident to me that this model would be useful in the classification of hydrocephalus and formed the basis of the book chapter that was produced $[13,15]$.

Review of the inconsistencies in the criteria that were being used for the selection of patients for endoscopic third ventriculostomy led to the unassailable conclusion that simply classifying hydrocephalus as communicating or non-communicating at a time when the actual point of the obstruction or restriction of CSF flow could be determined in most cases had become inadequate. Other questions that remained to be answered and could not be using the simple classification of Dandy included why some patients who developed hydrocephalus in infancy or had arachnoid cysts treated with shunts in infancy develop severely increased intracranial pressure with no expansion of the ventricles or cyst at the time of shunt failure. A more sophisticated 
Fig. 1 Multicompartmental model of ventricular volume regulation. Used with permission from Karger (Rekate et al. 1988) a

0 if $\mathrm{P}_{\mathrm{C}}<\mathrm{PO}_{\mathrm{O}}$

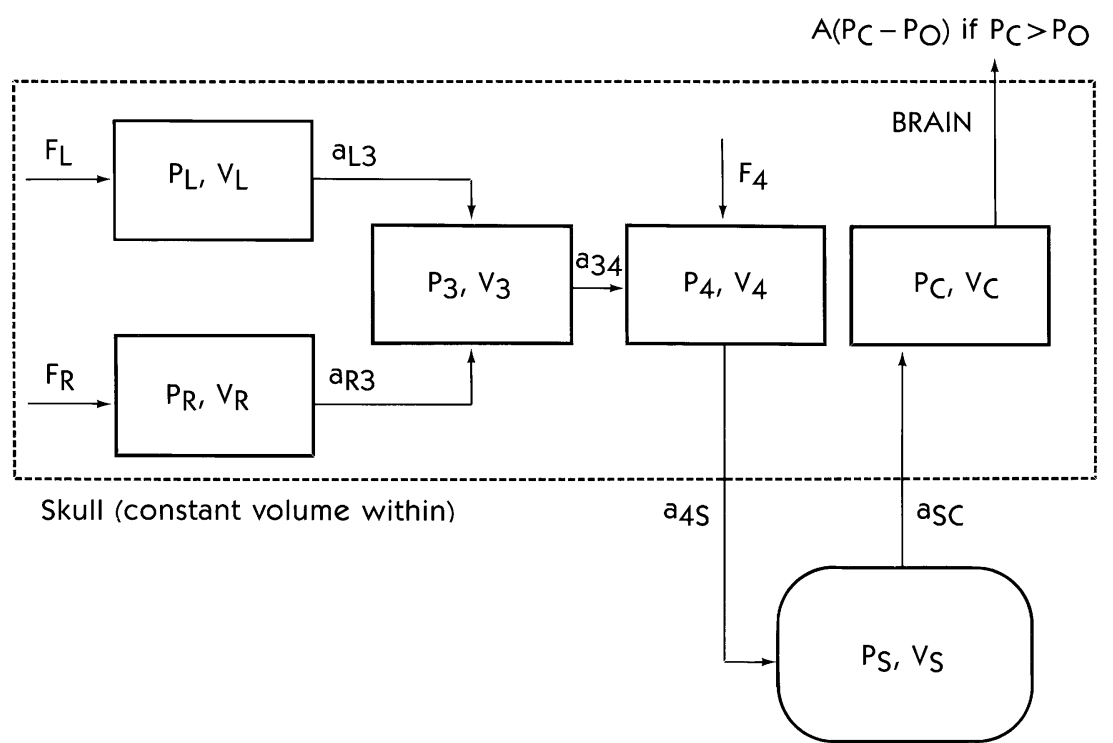

b

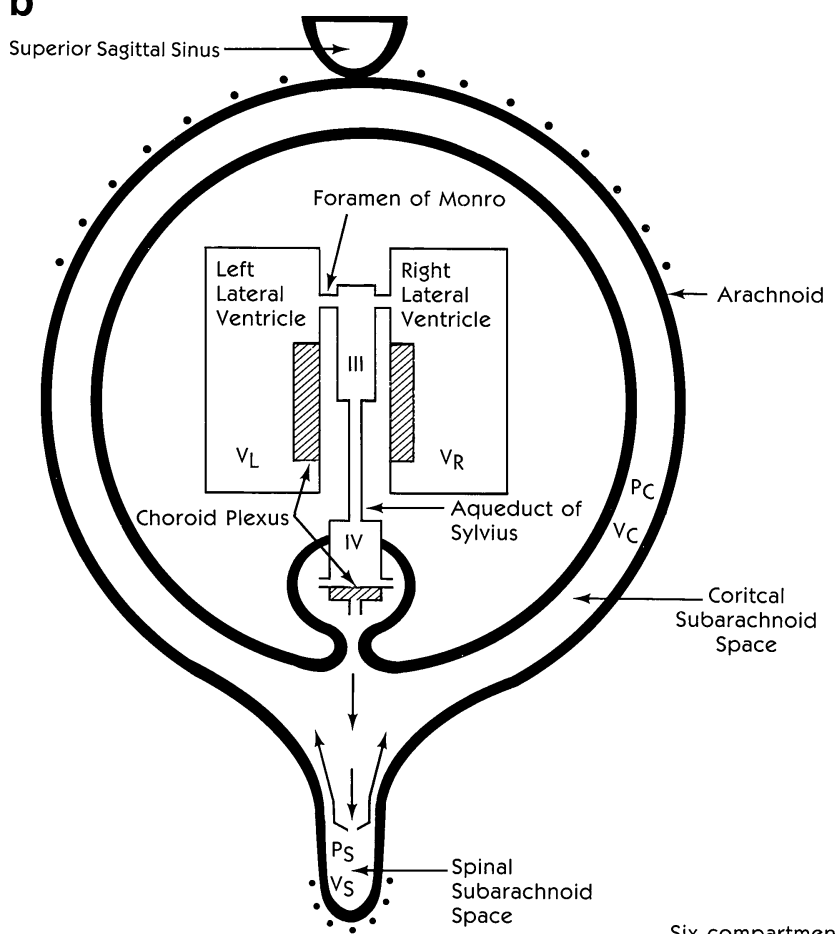

Six compartment model of CSF pathways classification was now available but not generally known or accepted. Techniques for the study of CSF dynamics had improved dramatically since the studies of Dandy. Tools such as magnetic resonance imaging (MRI), Cine MRI flow studies, cisternography utilizing dye studies and especially long-term studies of the outcomes of treatment decisions make it possible to accurately define a point of restriction of flow.

I was then invited at the inaugural meeting of the International Society for Hydrocephalus and CSF Research by meeting president Dr. Petra Klinge to give a talk on the subject of the definition and classification of hydrocephalus. In preparation for this talk I wrote an article published in the open access on-line journal Cerebrospinal Fluid Research entitled "A Contemporary Definition and Classification of Hydrocephalus: A Straw Man to Produce Debate." At the time of the presentation at the meeting and during the experience in Hannover, I called on the participants to challenge and discuss the classification [13].

Following the "roll-out" of the concepts a large number of recognized experts in hydrocephalus treatment and research were contacted, and two meetings were held. The 


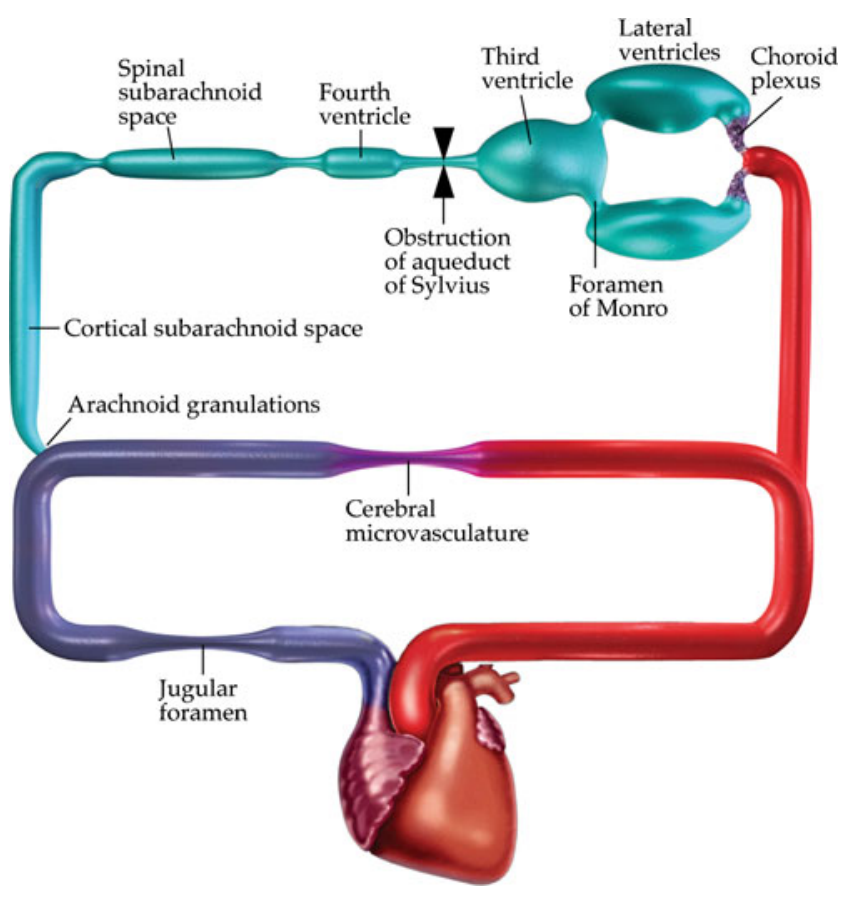

Fig. 2 Artist's concept of circuit diagram of cerebral blood flow and CSF production and flow. Used with permission from Barrow Neurological Institute (Rekate et al. 2008)

first occurred in Los Angeles at the 40th annual meeting of the International Society for Pediatric Neurosurgery. The second meeting was held in Phoenix in January of 2010. The participants in the process of producing a consensus statement are listed in the addendum at the end of this discussion ("Appendix"). At the end of these discussions a consensus was reached and agreed to by all. The results of these meetings were presented at the International Hydrocephalus Symposium in Crete in May 2010 and at the International Society for Research in Hydrocephalus and Spina Bifida in June as well as the Hydrocephalus Association Convention in July of that year. There was widespread support for the classification and enthusiasm that it would serve as a platform for research and structure to analyze research using animal models.

The structure of the consensus was that the first level of classification of hydrocephalus would be based on the point where the flow of CSF is restricted. The potential sites of restriction of flow as seen in Fig. 2 would be the foramina of Monro, the aqueduct of Sylvius, the basal cisterns, the arachnoid granulations, and outflow of venous blood from the dural venous sinuses. It was also recognized that hydrocephalus could exist and progress without a point of obstruction or increased resistance to flow. This would truly be communicating hydrocephalus.

The primary classification of point of obstruction would then be modified by the etiology of the inciting condition, the chronicity or rapidity of onset, and the age of the person or experimental animal. It is expected that the point of obstruction will be sought in both experimental animals as well as patients in clinical studies so that likes can be compared.

Exploring the hydraulic circuit using animal models

The collaboration between the Division of Neurosurgery, the department of Systems and Design Engineering and the Electronics Design Center at Case Western Reserve University began with weekly meetings for several hours in which engineering and physiological concepts were discussed and important literature researched. The plan was to develop a mathematical model of ventricular volume regulation and cerebrospinal fluid (CSF) dynamics which would include actually measured parameters such as starting CSF volumes, intracranial pressures (ICP) and rate of CSF production as well as presumed but so far unmeasured parameters such as the resistance elements within the CSF system such as the resistance at the aqueduct of Sylvius. We would then manipulate the model to see what changes would occur in predicted volumes and pressures of the CSF compartments. Initially the expectation was that intracranial pulsation would play a major role in the process, but at that time there was no model available that could be used to add an alternating current (AC) circuit to the model. We decided that we would begin by testing the issues related to the bulk flow model and would move on to the AC or pulse-wave model at a later time.

The model thus created included a fixed volume of the intracranial compartment, a spinal compartment that was outside the fixed volume constraint and six CSF compartments and the brain and spinal cord dealt with as a single entity. It was essential that the research be able to study and lead to the understanding of not only hydrocephalus but also normal pressure hydrocephalus $(\mathrm{NPH})$ and pseudotumor cerebri (PC) $[10,11]$.

\section{Resistance elements within the CSF pathways}

The first step in the experimental design here was to actually measure the significance of the proposed resistance elements within the CSF pathways. Utilizing a canine model of hydrocephalus and with normal controls, we instrumented the compartments to measure the pressure differentials across various presumed points of obstruction. We cannulated the lateral ventricles, third ventricle and cisterna magna in both normal and hydrocephalic dogs and infused artificial CSF into one of the lateral ventricles while measuring the pressure in the various components. In these experiments we found that no pressure differential could be measured anywhere in the system. This inability to measure pressure 
differentials within the intracranial compartment also was found when there was a known point of obstruction in the hydrocephalic animals.

We then implanted a balloon in the cortical subarachnoid space of the dog and applied a wave form to it with multiple ICP transducers intracranially and found that the pulse wave was transmitted undiminished and instantaneously to all transducers intracranially [16]. While one of the potential explanations is that our transducers which were both strain gauge and fluid-coupled types were not sufficiently sensitive to measure the pressure differentials. They had a sensitivity of plus or minus $1 \mathrm{mmHg}$. The alternative explanation and the one that is most defensible is that the brain is a viscoelastic substance and in this set of experiments was acting as a fluid chamber where changes in pressure are transmitted instantaneously and fully to all areas.

Measurable pressure differentials were found in only one context. If one of the lateral ventricles is drained to a subatmospheric pressure, a pressure differential of $12 \mathrm{mmHg}$ can be accurately measured [17]. This condition mirrors the situation of "post-shunt ventricular asymmetry" seen in children who are shunted and have an intact septum pellucidum whose shunted ventricle is uniformly smaller than the contralateral ventricle [18, 19]. Anatomic studies of the foramen of Monro have shown that this phenomenon is due to the fact that the septum pellucidum is drawn toward the shunted or drained ventricle and comes to rest on the head of the caudate nucleus leading to a functional and reversible obstruction to flow [17].

The intracisternal kaolin model utilized in our experiments led to hydrocephalus by obstructing the outflow of
CSF from the outlet foramina of the fourth ventricle. The condition was found using ventriculography to be accompanied by, in nearly all animals, syringomyelia as well making this a model of what Oi would call hydromyelic hydrocephalus [20].

Obstruction between the spinal and cortical subarachnoid spaces is rarely diagnosed but is probably the cause of many or most cases of NPH [21, 22]. Older studies utilizing protein labeled with a radioisotope tracer showed that the dye injected into the spinal subarachnoid space in patients quickly entered the ventricles but its clearance into the cortical subarachnoid space was dramatically delayed suggesting a blockage between the SSAS and CSAS. Di Rocco performed autopsies on patients who had responded to shunting for NPH and who died subsequently of other causes. He found dense arachnoidal thickening around the brainstem in the posterior fossa [21].

McAllister has studied this extraventricular obstructive hydrocephalus using a rat model of hydrocephalus where the kaolin is injected through the skull base into the lower CSAS. Hydrocephalus in these animals develops quite slowly and is usually mild but does occur [23].

Blockage between the SSAS and CSAS results from either subarachnoid hemorrhage or infection. It frequently involves the area around the brainstem selectively, and this form of hydrocephalus has been shown to be amenable to endoscopic third ventriculostomy [24]. This point of obstruction is also the explanation for the successful management of NPH utilizing endoscopic third ventriculostomy (ETV) [25].
Table 1 Utility of point of obstruction model

$D X$ diagnosis, ETV endoscopic third ventriculostomy, CSAS cortical subarachnoid space, $L P$ lumboperitoneal, $V P$ ventriculoperitoneal

\begin{tabular}{lll}
\hline Point of obstruction & Differential DX & Treatments available \\
\hline Foramen of Monro & Tumor & Shunt (unilateral or bilateral) \\
& Congenital absence & Endoscopic septum opening \\
& Ventriculitis & \\
& Functional & \\
Aqueduct of Sylvius & Tumor & Shunt \\
& Birth defect & ETV \\
& Secondary & \\
4th Ventricle foramina & Infection & Shunt \\
& Tumor & ETV \\
& Severe Chiari & Surgical opening \\
Spinal to CSAS & Subarachnoid & Shunt \\
& Hemorrhage & ETV \\
Arachnoid villi & LP shunt & \\
Venous hypertension & Hemorrhage or infection & VP or LP shunt \\
& Pseudotumor & VP or LP shunt \\
& Congenital hydrocephalus & Clot lysis \\
& Sinus thrombosis & Bariatric surgery for obesity-related pseudotumor \\
\hline
\end{tabular}


Venous hypertension causing hydrocephalus

Increased pressure in the dural venous sinuses that occurs in adults results in PC and not hydrocephalus [26]. Drainage of CSF into the dural venous sinuses requires a gradient between the ICP and sagittal sinus pressure of 5-7 mmHg [27, 28]. If the pressure in the sagittal sinus is elevated, the ICP must elevate as well in order for CSF to be absorbed. If the volume of the skull is fixed, the ICP goes up until CSF can be absorbed. If on the other hand the skull is not of a fixed volume, the ICP is in communication with atmospheric pressure. This occurs in the case of small babies and in the case of patients undergoing large craniectomies for stroke or trauma. In this situation the ICP cannot go above the atmospheric pressure, and the patient develops hydrocephalus.

Exploring this phenomenon, Olivero occluded the superior sagittal sinus of normal and craniectomized rabbits. The rabbits whose skull was intact developed intracranial hypertension without ventriculomegaly. The craniectomized rabbits however developed hydrocephalus [29].

\section{True communicating hydrocephalus: Hydrocephalus without a point of obstruction}

Much of my fascination with the study of the physics of CSF and hydrocephalus was stimulated by the experiments of Di Rocco and colleagues who produced hydrocephalus in experimental animals by implanting a balloon in the ventricle and augmenting the pulse wave. There was no source of obstruction to the flow of CSF in these animals [30-32].

In order to study whether it was possible to produce hydrocephalus in experimental animals without a point of obstruction and without the augmentation of the pulsation, we did prolonged infusions of artificial CSF into normal and hydrocephalic dogs to simulate the overproduction of CSF by choroid plexus papillomas. In these experiments the normal dogs did develop modest ventriculomegaly, but the dogs that had been previously made hydrocephalic with kaolin developed severe ventriculomegaly [33].

\section{Clinical utility of the classification scheme}

As opposed to Dandy, we now have the ability to actually define the specific point of obstruction to the flow of CSF within the craniospinal axis utilizing MRI sequences and the injection of iodine dye or radioactive tracers. This information is valuable both in deciding among different pathologic states in the differential diagnosis as well as deciding among different treatment options. Table 1 outlines the potential diagnoses and the treatment options related to the various points of obstruction to the hydraulic circuit. This classification should prove useful in future experimental design, assessment of unexpected results of treatment of hydrocephalus and the design of prospective trials for the treatment of subtypes of hydrocephalus.

Acknowledgments Much of the basic science research presented here was supported by a grant from the National Institutes of Health (RO1 NS22901). The process of developing the concensus on the classification of hydrocephalus was supported by a gift from the Hecht-Levi Foundation to support hydrocephalus research.

Open Access This article is distributed under the terms of the Creative Commons Attribution Noncommercial License which permits any noncommercial use, distribution, and reproduction in any medium, provided the original author(s) and source are credited.

\section{Appendix}

Members of the Hydrocephalus Classification Study Group

Osamu Sato MD Tokyo Japan

Shizuo Oi MD, PhD Tokyo, Japan

Charles Teo MD Sydney, Australia

John Pickard MD Cambridge, UK

Marion Walker MD Salt Lake City, UT

J. Patrick McAllister PhD Salt Lake City, UT

Gordon McComb MD Los Angeles, CA

Martina Messing-Jùnger MD Sankt Augustin, Germany

Michael Pollay MD Sun City West, AZ

Spyros Sgouros MD Athens, Greece

Petra Klinge MD, PhD Providence, RI

Thomas Brinker MD, PhD Providence, RI

Conrad Johansson PhD Providence, RI

Concezio Di Rocco MD Rome, Italy

Harold L. Rekate MD Great Neck, NY

\section{References}

1. Pudenz RH (1981) The surgical treatment of hydrocephalus - an historical review. Surg Neurol 15:15-26

2. Dandy WE (1919) Fluoroscopy of the cerebral ventricles. Bull Johns Hopkins Hosp 30:29-33

3. Diseases of the central nervous system and sense organs. ICD-9CM 9th revision. Eds. Hart AC, Stegman MS, Ford B, et al. Ingenix, 2010. 90

4. Raimondi AJ (1994) A unifying theory for the definition and classification of hydrocephalus. Childs Nerv Syst 10:2-12

5. Mori K (1995) Current concept of hydrocephalus: evolution of new classifications. Childs Nerv Sys 11(9):523-531

6. Beni-Adani L, Biani N, Ben-Sirah L, Constantini S (2006) The occurrence of obstructive vs absorptive hydrocephalus in 
newborns and infants: relevance to treatment choices. Childs Nerv Syst 22(12): 1543-1563

7. Oi S, Di Rocco C (2006) Proposal of "evolution theory in cerebrospinal fluid dynamics" and minor pathway hydrocephalus in developing immature brain. Childs Nerv Syst 22(7):662-669

8. Rekate H (2000) Hydrocephalus: classification and pathophysiology. In: McLone D (ed) Pediatric neurosurgery: surgery of the developing nervous system, 4th edn. Saunders, Philadelphia, pp 253-295

9. Spertell RB (1980) The response of brain to transient elevations in intraventricular pressure. J Neurol Sci 48(3):343-352

10. Rekate HL (1994) The usefulness of mathematical modeling in hydrocephalus research. Childs Nerv Syst 10(1):13-18

11. Rekate HL, Brodkey JA, Chizeck HJ et al (1988) Ventricular volume regulation: a mathematical model and computer simulation. Pediatr Neurosci 14(2):77-84

12. Rekate HL (1989) Circuit diagram of the circulation of cerebrospinal fluid. Pediatr Neurosurg 21(4):248-252

13. Rekate HL (2008) The definition and classification of hydrocephalus: a personal recommendation to stimulate debate. Cerebrospinal Fluid Res 5(2):2

14. Rekate HL (1996) Cerebrospinal fluid circulation. In: Guthkelch $\mathrm{AN}$, Misulis KE (eds) Scientific foundations of neurology. Blackwell, Cambridge, pp 323-336

15. Rekate HL, Nadkarni TD, Wallace D (2008) The importance of the cortical subarachnoid space in understanding hydrocephalus. J Neurosurg Pediatr 2:1-11

16. Rekate HL, McCormick JM, Ko W (1990) Failure to demonstrate a brain transmissibility factor. In: Marlin AE (ed) Concepts in pediatric neurosurgery, 10th edn. Karger, Basel, pp 235-242

17. Rekate HL, Williams FC, Brodkey JA et al (1988) Resistance of the foramen of Monro. Pediatr Neurosci 14(2):85-89

18. Kaufman B, Weiss MH, Young HF, Nulsen FE (1973) Effects of prolonged cerebrospinal fluid shunting on the skull and brain. $\mathrm{J}$ Neurosurg 38(3):288-297

19. Linder M, Diehl JT, Sklar FA (1981) Significance of postshunt ventricular asymmetries. J Neurosurg 55(2):183-186

20. Oi S, Kudo H, Yamada H et al (1991) Hydromyelic hydrocephalus. Correlation of hydromyelia with various stages of hydrocephalus in postshunt isolated compartments. J Neurosurg 74(3):371-379

21. Di Rocco C, di Tripani G, Maira G, Bentvoglio M, Macchi G, Rossi GF (1977) Anatomo-clinical correlations in normotensive hydrocephalus. Reports on three cases. J Neurol Sci 33(3):437452

22. Jellinger K (1976) Neuropathological aspects of dementias resulting from abnormal blood and cerebrospinal fluid dynamics. Acta Neurol Belg 76(2):83-102

23. Nagra G, Li J, McAllister JP, Miller J, Wagshul M, Johnston M (2008) Impaired lymphatic cerebrospinal fluid absorption in a rat model of kaolin-induced communicating hydrocephalus. Am J Physiol Regul Integr Comp Physiol 294(5):R1752-R1759

24. Siomin V, Cinalli G, Grotenhuis A et al (2002) Endoscopic third ventriculostomy in patients with cerebrospinal fluid infection and/ or hemorrhage. J Neurosurg 97(3):519-524

25. Gangemi M, Maiuri F, Naddeo M et al (2008) Endoscopic third ventriculostomy in idiopathic normal pressure hydrocephalus: an Italian multicenter study. Neurosurgery 63(1):62-67

26. Karahalios DG, Rekate HL, Khayata MH, Apostolides PJ (1996) Elevated intracranial venous pressure as a universal mechanism in pseudotumor cerebri of varying etiologies. Neurology 46(1):198-202

27. McCormick JM, Yamada K, Rekate HL, Miyaki H (1992) Time course of intraventricular pressure change in a canine model of hydrocephalus: its relationship to sagittal sinus elastance. Pediatr Neurosurg 18(3):127-133

28. Olivero WC, Rekate HL, Chizeck HJ et al (1988) Relationship between intracranial and sagittal sinus pressure in normal and hydrocephalic dogs. Pediatr Neurosci 14(4):196-201

29. Olivero WC, Asner N (1992) Occlusion of the sagittal sinus in craniectomized rabbits. Childs Nerv Syst 8(6):307-309

30. Di Rocco C, di Tripani G, Pettorossi VE, Caldarelli M (1979) On the pathology of experimental hydrocephalus induced by artificial increase in endoventricular CSF pulse pressure. Childs Brain 5 (2):81-95

31. Di Rocco C, Pettorossi VE, Caldarelli M, Mancinelli R, Velardi F (1977) Experimental hydrocephalus following mechanical increment of intraventricular pulse pressure. Experientia 33(11):1470-1472

32. Di Rocco C, Pettorossi VE, Caldarelli M, Mancinelli R, Velardi F (1978) Communicating hydrocephalus induced by mechanically increased amplitude of the intraventricular cerebrospinal fluid pressure: experimental studies. Exp Neurol 59(1):40-52

33. Rekate HL, Erwood S, Brodkey JA et al (1985) Etiology of ventriculomegaly in choroid plexus papilloma. Pediatr Neurosci 12(4-5):196-201 